УДК 517.9

O. G. Nakonechnyi, P. M. Zinko, I. M. Shevchuk

\title{
ANALYSIS THE SOLUTIONS OF THE DIFFERENTIAL NON-LINEAR EQUATIONS DESCRIBING THE INFORMATION SPREADING PROCESS WITH JUMP DISCONTINUITY
}

\begin{abstract}
O. G. Nakonechnyi, P. M. Zinko, I. M. Shevchuk. Analysis the solutions of the differential nonlinear equations describing the information spreading process with jump discontinuity, Mat. Stud. 51 (2019), 159-167.

In this paper, we introduce a mathematical model of spreading any type of information. The model has the form of a system of nonlinear differential equations with non-stationary parameters. We have suggested the explicit solutions of the system differential non-linear equations describing the information spreading process. A special case of this model with jump discontinuity is considered. The numerical experiments demonstrated the practical meaning of the offered results. The results can be useful for algorithm development for estimation of dynamic of information spreading process.
\end{abstract}

1. Introduction. The mathematical apparatus of the theory of differential equations (in particular [1], [2]) is often used to model socio-communicative processes (for example [3]-[5]). One of the ways of presenting the information spreading process in society is the system of nonlinear differential equations.

Let us consider a certain social community of $L$ people. Suppose, a community is influenced by one of $N$ sources of information at some point in time $t \in\left(t_{0}, T\right)$. The numbers of people who have accepted the information messages from $k$-th information flow $(k=$ $=\overline{1, N})$ depends on external influence and interpersonal communication. Let us denote $x_{k}(t)$, $k=\overline{1, N}, t \in\left(t_{0}, T\right)$ are the numbers of people who have accepted the information messages from $k$-th information flow $(k=\overline{1, N})$ at point of time $t ; b_{k}(t), k=\overline{1, N}, t \in\left(t_{0}, T\right)$ are the intensities of communication, $u_{k}(t), k=\overline{1, N}, t \in\left(t_{0}, T\right)$ are external influences. Time variable of values $x_{k}(t), k=\overline{1, N}, t \in\left(t_{0}, T\right)$ we describe by differential equation system

$$
\dot{x}_{k}(t)=b_{k}(t) x_{k}(t)\left(L-\sum_{i=1}^{N} x_{i}(t)\right)+u_{k}(t), t \in(0, T), k=\overline{1, N}
$$

with initial conditions

$$
x_{k}\left(t_{0}\right)=x_{k}^{0}, k=\overline{1, N} .
$$

Works [6] are devoted to analysis of solution oh this model (1), (2) with stationary parameters and the special type of $u_{k}(t), k=\overline{1, N}, t \in\left(t_{0}, T\right)$. Conditions of existence of

2010 Mathematics Subject Classification: 34A05.

Keywords: non-linear differential equation; stationary and non-stationary parameters; mathematical model of information spreading process; explicit solution.

doi:10.15330/ms.51.2.159-168

(C) O. G. Nakonechnyi, P. M. Zinko, I. M. Shevchuk , 2019 
range of first-approximation stability of the solutions are considered in [7], [8]. A separate practical-important case of the model $(1),(2)$ is the equations with jumps discontinuity that are appropriate to be modeled as pulsed perturbations (for example, [9]).

2. Analysis of the basis model of information spreading process. Now let us take a detailed look at the model (1) and the external influences are simulated us

$$
u_{k}(t)=a_{k}(t)\left(L-\sum_{i=1}^{N} x_{i}(t)\right), k=\overline{1, N}, t \in\left(t_{0}, T\right) .
$$

Then model (1), (2) has the following form

$$
\dot{x}_{k}(t)=\left(a_{k}(t)+b_{k}(t) x_{k}(t)\right)\left(L-\sum_{i=1}^{N} x_{i}(t)\right), t \in\left(t_{0}, T\right), x_{k}\left(t_{0}\right)=x_{k}^{0}, k=\overline{1, N} .
$$

Theorem 1. Let the conditions $b(t)=b_{k}(t), k=\overline{1, N}, t \in\left(t_{0}, T\right)$ are satisfied for the system (3). Than the solutions $x_{k}(t), k=\overline{1, N}, t \in\left(t_{0}, T\right)$ are calculated by the formulas

$$
\begin{gathered}
x_{k}(t)=x_{k}^{0} \exp \left\{\int_{t_{0}}^{t} b(s) y(s) d s\right\}+ \\
+\int_{t_{0}}^{t} a_{k}(\tau) y(\tau) \exp \left\{\int_{\tau}^{t} b(s) y(s) d s\right\} d \tau, t \in\left(t_{0}, T\right), k=\overline{1, N},
\end{gathered}
$$

where

$$
\begin{gathered}
y(t)=\frac{y\left(t_{0}\right)}{\exp \left\{-\int_{t_{0}}^{t} a(s) d s\right\}+y\left(t_{0}\right) \int_{t_{0}}^{t} b(\tau) \exp \left\{-\int_{\tau}^{t} a(s) d s\right\} d \tau}, t \in\left(t_{0}, T\right), \\
a(t)=\sum_{i=1}^{N} a_{i}(t)+b(t) L, t \in\left(t_{0}, T\right), y\left(t_{0}\right)=L-\sum_{k=1}^{N} x_{k}^{0} .
\end{gathered}
$$

Proof. Let us denote $y(t)=L-\sum_{k=1}^{N} x_{k}(t), t \in\left(t_{0}, T\right)$. Therefore the next equalities are fulfilled

$$
\begin{aligned}
\dot{y}(t)= & -\sum_{k=1}^{N} \dot{x}_{k}(t)=-\sum_{k=1}^{N}\left(a_{k}(t)+b(t) x_{k}(t)\right)\left(L-\sum_{i=1}^{N} x_{i}(t)\right)= \\
= & -\left(\sum_{k=1}^{N} a_{k}(t)+b(t) \sum_{k=1}^{N} x_{k}(t)\right)\left(L-\sum_{i=1}^{N} x_{i}(t)\right)= \\
& =\left(\sum_{k=1}^{N} a_{k}(t)+b(t)(L-y(t))\right) y(t), \quad t \in\left(t_{0}, T\right) .
\end{aligned}
$$

The function $y(t), t \in\left(t_{0}, T\right)$ has the form of Riccati equation

$$
\dot{y}(t)=(a(t)-b(t) y(t)) y(t), t \in\left(t_{0}, T\right)
$$

with the initial condition

$$
y\left(t_{0}\right)=L-\sum_{k=1}^{N} x_{k}^{0}
$$


Denote the function $z(t)=(y(t))^{-1}, t \in\left(t_{0}, T\right)$ and this function satisfies representation $\dot{z}(t)=-(y(t))^{-2} \dot{y}(t), t \in\left(t_{0}, T\right)$.

Myltiplying the left-hand and the right-hand side of (5) by $\left(-y^{-2}(t)\right)$ we have the formula

$$
-\frac{1}{y^{2}}(t) \dot{y}(t)=b(t)-\frac{a(t)}{y(t)}, t \in\left(t_{0}, T\right) .
$$

Instead of differential equation (6) we have Cauchy's problem for the function $z(t), t \in$ $\left(t_{0}, T\right)$

$$
\dot{z}(t)=b(t)-a(t) z(t), \quad t \in\left(t_{0}, T\right), z\left(t_{0}\right)=y^{-1}\left(t_{0}\right) .
$$

On the basis of the Cauchy formula the equation (7) has the solution

$$
z(t)=z\left(t_{0}\right) \exp \left\{-\int_{0}^{t} a(s) d s\right\}+\int_{0}^{t} b(\tau) \exp \left\{-\int_{\tau}^{t} a(s) d s\right\} d \tau, \quad t \in\left(t_{0}, T\right) .
$$

Since the equality satisfies

$$
y(t)=(z(t))^{-1}, t \in\left(t_{0}, T\right)
$$

we get the next expression from (8)

$$
y(t)=\frac{y\left(t_{0}\right)}{\exp \left\{-\int_{t_{0}}^{t} a(s) d s\right\}+y\left(t_{0}\right) \int_{t_{0}}^{t} b(\tau) \exp \left\{-\int_{\tau}^{t} a(s) d s\right\} d \tau}, \quad t \in(0, T) .
$$

The equation (3) can be written as

$$
\dot{x}_{k}(t)=\left(a_{k}(t)+b(t) x_{k}(t)\right) y(t), t \in\left(t_{0}, T\right), k=\overline{1, N}
$$

with the initial conditions

$$
x_{k}\left(t_{0}\right)=x_{k}^{0}, k=\overline{1, N} .
$$

On the basis of the Cauchy formula the solutions of the system (10) are calculated as

$$
\begin{gathered}
x_{k}(t)=x_{k}^{0} \exp \left\{\int_{t_{0}}^{t} b(s) y(s) d s\right\}+ \\
+\int_{t_{0}}^{t} a_{k}(\tau) y(\tau) \exp \left\{\int_{\tau}^{t} b(s) y(s) d s\right\} d \tau, t \in\left(t_{0}, T\right), k=\overline{1, N} .
\end{gathered}
$$

The theorem has been proven.

Theorem 2. Let the conditions $b=b_{k}(t), a_{k}=a_{k}(t), k=\overline{1, N}, a=a(t), t \in\left(t_{0}, T\right)$ be satisfied for the system (3). Than the functions $x_{k}(t), k=\overline{1, N}, t \in\left(t_{0}, T\right)$ are calculated as

$$
\begin{gathered}
x_{k}(t)=\left[\left(x_{k}^{0}\left(a-y\left(t_{0}\right) b\right)-a_{k} y\left(t_{0}\right)\right) \exp \left\{\left(t_{0}-t\right) a\right\}+y\left(t_{0}\right)\left(x_{k}^{0} b+a_{k}\right)\right] \times \\
\times\left(a \exp \left\{\left(t_{0}-t\right) a\right\}\right)^{-1}, t \in\left(t_{0}, T\right), k=\overline{1, N} .
\end{gathered}
$$

Proof. Since the parameters $b, a_{k}, k=\overline{1, N}$ of the system (3) are stationary the equality (9) take the form

$$
y(t)=\frac{y\left(t_{0}\right)}{\exp \left\{-\int_{t_{0}}^{t} a d s\right\}+y\left(t_{0}\right) \int_{t_{0}}^{t} b \exp \left\{-\int_{\tau}^{t} a d s\right\} d \tau}, t \in\left(t_{0}, T\right) .
$$


The representation (12) can be written as

$$
\begin{gathered}
y(t)=\frac{y\left(t_{0}\right)}{\exp \left\{\left(t_{0}-t\right) a\right\}+y\left(t_{0}\right) \int_{t_{0}}^{t} b \exp \{(\tau-t) a\} d \tau}= \\
=\frac{y\left(t_{0}\right)}{\exp \left\{\left(t_{0}-t\right) a\right\}+\frac{y\left(t_{0}\right) b}{a} \exp \{-a t\}\left(\exp \{a t\}-\exp \left\{a t_{0}\right\}\right)}= \\
=\frac{a y\left(t_{0}\right)}{\left(a-y\left(t_{0}\right) b\right) \exp \left\{\left(t_{0}-t\right) a\right\}+y\left(t_{0}\right) b}, \quad t \in\left(t_{0}, T\right) .
\end{gathered}
$$

In this case the equations (4) are presented in the following form

$$
\begin{gathered}
x_{k}(t)=x_{k}^{0} \exp \left\{b \int_{t_{0}}^{t} y(s) d s\right\}+ \\
+a_{k} \int_{t_{0}}^{t} y(\tau) \exp \left\{b \int_{\tau}^{t} y(s) d s\right\} d \tau, \quad t \in\left(t_{0}, T\right), \quad k=\overline{1, N} .
\end{gathered}
$$

We have the expression

$$
\begin{gathered}
\int_{\tau}^{t} y(s) d s=\int_{\tau}^{t} \frac{a y\left(t_{0}\right) d s}{\left(a-y\left(t_{0}\right) b\right) \exp \left\{\left(t_{0}-s\right) a\right\}+y\left(t_{0}\right) b}= \\
=-y\left(t_{0}\right) \int_{\tau}^{t} \frac{d\left(\exp \left\{\left(t_{0}-s\right) a\right\}\right)}{\exp \left\{\left(t_{0}-s\right) a\right\}\left(\left(a-y\left(t_{0}\right) b\right) \exp \left\{\left(t_{0}-s\right) a\right\}+y\left(t_{0}\right) b\right)}= \\
=\frac{1}{b} \int_{\tau}^{t} \frac{d\left(\left(a-y\left(t_{0}\right) b\right) \exp \left\{\left(t_{0}-s\right) a\right\}\right)}{\left(a-y\left(t_{0}\right) b\right) \exp \left\{\left(t_{0}-s\right) a\right\}+y\left(t_{0}\right) b}-\int_{\tau}^{t} \frac{d\left(\exp \left\{\left(t_{0}-s\right) a\right\}\right)}{b \exp \left\{\left(t_{0}-s\right) a\right\}}= \\
=\frac{1}{b}\left(\ln \left|\left(a-y\left(t_{0}\right) b\right) \exp \left\{\left(t_{0}-s\right) a\right\}+y\left(t_{0}\right) b\right|_{\tau}^{t}-\ln \left|\exp \left\{\left(t_{0}-s\right) a\right\}\right|_{\tau}^{t}\right)= \\
=\ln \left|\frac{\left(\left(a-y\left(t_{0}\right) b\right) \exp \left\{\left(t_{0}-t\right) a\right\}+y\left(t_{0}\right) b\right) \exp \left\{\left(t_{0}-\tau\right) a\right\}}{\exp \left\{\left(t_{0}-t\right) a\right\}\left(\left(a-y\left(t_{0}\right) b\right) \exp \left\{\left(t_{0}-\tau\right) a\right\}+y\left(t_{0}\right) b\right)}\right|^{\frac{1}{b}}, t, \tau \in\left(t_{0}, T\right),
\end{gathered}
$$

thus the formula (13) can be represented as

$$
\begin{gathered}
x_{k}(t)=x_{k}^{0} \exp \left\{b \ln \left|\frac{\left(a-y\left(t_{0}\right) b\right) \exp \left\{\left(t_{0}-t\right) a\right\}+y\left(t_{0}\right) b}{a \exp \left\{\left(t_{0}-t\right) a\right\}}\right|^{\frac{1}{b}}\right\}+ \\
+a_{k} \int_{t_{0}}^{t} \frac{a y\left(t_{0}\right)}{\left(a-y\left(t_{0}\right) b\right) \exp \{(t-\tau) a\}+y\left(t_{0}\right) b} \times \\
\times \exp \left\{b \ln \left|\frac{\left(\left(a-y\left(t_{0}\right) b\right) \exp \left\{\left(t_{0}-t\right) a\right\}+y\left(t_{0}\right) b\right) \exp \left\{\left(t_{0}-\tau\right) a\right\}}{\exp \left\{\left(t_{0}-t\right) a\right\}\left(\left(a-y\left(t_{0}\right) b\right) \exp \left\{\left(t_{0}-\tau\right) a\right\}+y\left(t_{0}\right) b\right)}\right| \frac{1}{b}\right\}= \\
=x_{k}^{0}\left|\frac{\left(a-y\left(t_{0}\right) b\right) \exp \left\{\left(t_{0}-t\right) a\right\}+y\left(t_{0}\right) b}{a \exp \left\{\left(t_{0}-t\right) a\right\}}\right|+ \\
+a_{k} a y\left(t_{0}\right) \frac{\left(a-y\left(t_{0}\right) b\right) \exp \left\{\left(t_{0}-t\right) a\right\}+y\left(t_{0}\right) b}{\exp \left\{\left(t_{0}-t\right) a\right\}} \times \\
\times \int_{t_{0}}^{t} \frac{\exp \left\{\left(t_{0}-\tau\right) a\right\} d \tau}{\left(\left(a-y\left(t_{0}\right) b\right) \exp \left\{\left(t_{0}-\tau\right) a\right\}+y\left(t_{0}\right) b\right)^{2}}, \quad t \in\left(t_{0}, T\right), k=\overline{1, N} .
\end{gathered}
$$

The next expressions satisfied

$$
\int_{t_{0}}^{t} \frac{\exp \left\{\left(t_{0}-\tau\right) a\right\} d \tau}{\left(\left(a-y\left(t_{0}\right) b\right) \exp \left\{\left(t_{0}-\tau\right) a\right\}+y\left(t_{0}\right) b\right)^{2}}=
$$




$$
\begin{gathered}
=-\frac{1}{a\left(a-y\left(t_{0}\right) b\right)} \int_{t_{0}}^{t} \frac{-a\left(a-y\left(t_{0}\right) b\right) \exp \left\{a\left(t_{0}-\tau\right) a\right\} d \tau}{\left(\left(a-y\left(t_{0}\right) b\right) \exp \left\{\left(t_{0}-\tau\right) a\right\}+y\left(t_{0}\right) b\right)^{2}}= \\
=\left.\frac{1}{a\left(a-y\left(t_{0}\right) b\right)} \frac{1}{\left(a-y\left(t_{0}\right) b\right) \exp \left\{\left(t_{0}-\tau\right) a\right\}+y\left(t_{0}\right) b}\right|_{t_{0}} ^{t}= \\
=\frac{1}{a\left(a-y\left(t_{0}\right) b\right)}\left(\frac{1}{\left(a-y\left(t_{0}\right) b\right) \exp \left\{\left(t_{0}-t\right) a\right\}+y\left(t_{0}\right) b}-\frac{1}{a}\right)= \\
=\frac{1}{a^{2}} \frac{1-\exp \left\{\left(t_{0}-t\right) a\right\}}{\left(a-y\left(t_{0}\right) b\right) \exp \left\{\left(t_{0}-t\right) a\right\}+y\left(t_{0}\right) b}, t \in\left(t_{0}, T\right) .
\end{gathered}
$$

Therefore the functions $x_{k}(t), k=\overline{1, N}, t \in(0, T)$ are found from the formulas

$$
\begin{gathered}
x_{k}(t)=x_{k}^{0}\left|\frac{\left(a-y\left(t_{0}\right) b\right) \exp \left\{\left(t_{0}-t\right) a\right\}+y\left(t_{0}\right) b}{a \exp \left\{\left(t_{0}-t\right) a\right\}}\right|+ \\
+a_{k} a y\left(t_{0}\right) \frac{\left(a-y\left(t_{0}\right) b\right) \exp \left\{\left(t_{0}-t\right) a\right\}+y\left(t_{0}\right) b}{\exp \left\{\left(t_{0}-t\right) a\right\}} \times \\
\times \frac{1}{a^{2}} \frac{1-\exp \left\{\left(t_{0}-t\right) a\right\}}{\left(a-y\left(t_{0}\right) b\right) \exp \left\{\left(t_{0}-t\right) a\right\}+y\left(t_{0}\right) b}= \\
=x_{k}^{0}\left|\frac{\left(a-y\left(t_{0}\right) b\right) \exp \left\{\left(t_{0}-t\right) a\right\}+y\left(t_{0}\right) b}{a \exp \left\{\left(t_{0}-t\right) a\right\}}\right|+\frac{a_{k} y\left(t_{0}\right)}{a} \frac{1-\exp \left\{\left(t_{0}-t\right) a\right\}}{\exp \left\{\left(t_{0}-t\right) a\right\}}= \\
=\frac{\left(x_{k}^{0}\left(a-y\left(t_{0}\right) b\right)-a_{k} y\left(t_{0}\right)\right) \exp \left\{\left(t_{0}-t\right) a\right\}+y\left(t_{0}\right)\left(x_{k}^{0} b+a_{k}\right)}{a \exp \left\{\left(t_{0}-t\right) a\right\}}, t \in\left(t_{0}, T\right), k=\overline{1, N} .
\end{gathered}
$$

3. Models of information spreading process with jump discontinuity. Consider the case of model (3) with a jump discontinuity in the time moments $t_{j}, t_{j} \in\left(t_{0}, T\right), j=\overline{1, M}$. Time variable of values $x_{k}(t), k=\overline{1, N}, t \in\left(t_{0}, T\right)$ are described by the system

$$
\left\{\begin{array}{l}
\dot{x}_{k}(t)=\left(a_{k}(t)+b_{k}(t) x_{k}(t)\right) \times \\
\times\left(L-\sum_{i=1}^{N} x_{i}(t)\right), t \in\left(t_{0}, T\right), t \neq t_{j}, \\
\left.\Delta x(t)\right|_{t=t_{j}}=x_{k}\left(t_{j}+0\right)-x_{k}\left(t_{j}-0\right)=\zeta_{j, k}, \\
x_{k}\left(t_{0}\right)=x_{k}^{0},
\end{array}\right.
$$

Theorem 3. Let the conditions $b(t)=b_{k}(t), t \in\left(t_{0}, T\right), k=\overline{1, N}$ be satisfied for the system (14). The functions $x_{k}(t), k=\overline{1, N}, t \in\left(t_{0}, t_{M+1}\right), t_{M+1}=T$ are calculated by the formulas

$$
\begin{gathered}
x_{k}(t)=\left[\ldots\left[x_{k}^{0} \exp \left\{\int_{t_{0}}^{t_{1}} b(s) y(s) d s\right\}+\int_{t_{0}}^{t_{1}} a_{k}(\tau) y(\tau) \exp \left\{\int_{\tau}^{t_{1}} b(s) y(s) d s\right\} d \tau+\zeta_{1, k}\right] \times\right. \\
\times \cdots \times \exp \left\{\int_{t_{j-2}}^{t_{j-1}} b(s) y(s) d s\right\}+\int_{t_{j-2}}^{t_{j-1}} a_{k}(\tau) y(\tau) \exp \left\{\int_{\tau}^{t_{j-1}} b(s) y(s) d s\right\} d \tau+ \\
\left.\quad+\zeta_{j-1, k}\right] \times \exp \left\{\int_{t_{j-1}}^{t} b(s) y(s) d s\right\}+ \\
+\int_{t_{j-1}}^{t} a_{k}(\tau) y(\tau) \exp \left\{\int_{\tau}^{t} b(s) y(s) d s\right\} d \tau, t \in\left(t_{j-1}, t_{j}\right), j=\overline{1, M+1}, k=\overline{1, N},
\end{gathered}
$$


where

$$
\begin{gathered}
y(t)=\left[\left(\left[\left(\ldots \left[y\left(t_{0}\right) \exp \left\{-\int_{t_{0}}^{t_{1}} a(s) d s\right\}+\right.\right.\right.\right.\right. \\
\left.\left.+\int_{t_{0}}^{t_{1}} b(\tau) \exp \left\{-\int_{\tau}^{t_{1}} a(s) d s\right\} d \tau\right]^{-1}-\sum_{k=1}^{N} \zeta_{1, k}\right) \times \ldots \times \\
\left.\left.\times \exp \left\{-\int_{t_{j-2}}^{t_{j-1}} a(s) d s\right\}+\int_{t_{j-2}}^{t_{j-1}} b(\tau) \exp \left\{-\int_{\tau}^{t_{j-1}} a(s) d s\right\} d \tau\right]^{-1}-\sum_{k=1}^{N} \zeta_{j-1, k}\right) \times \\
\left.\times \exp \left\{-\int_{t_{j-1}}^{t} a(s) d s\right\}+\int_{t_{j-1}}^{t} b(\tau) \exp \left\{-\int_{\tau}^{t} a(s) d s\right\} d \tau\right]^{-1}, t \in\left(t_{j-1}, t_{j}\right), j=\overline{1, M+1}, \\
y\left(t_{0}\right)=L-\sum_{k=1}^{N} x_{k}^{0}, a(t)=\sum_{i=1}^{N} a_{i}(t)+b(t) L, t \in\left(t_{0}, T\right) .
\end{gathered}
$$

Proof. In this case on the basis (4) the functions $x_{k}(t), t \in\left(t_{j-1}, t_{j}\right), j=\overline{1, M+1}, k=\overline{1, N}$ have the following form

$$
\begin{gathered}
x_{k}(t)=x_{k}\left(t_{j-1}\right) \exp \left\{\int_{t_{j-1}}^{t} b(s) y(s) d s\right\}+ \\
+\int_{t_{j-1}}^{t} a_{k}(\tau) y(\tau) \exp \left\{\int_{\tau}^{t} b(s) y(s) d s\right\} d \tau, t \in\left(t_{j-1}, t_{j}\right), k=\overline{1, N}, j=\overline{1, M+1},
\end{gathered}
$$

where

$$
x_{k}\left(t_{j-1}\right)=\left[\begin{array}{ll}
x_{k}^{0}, & j=1, \\
x_{k}\left(t_{j-1}-0\right)+\zeta_{j-1, k}, & j=\overline{2, M+1}, \quad k=\overline{1, N} .
\end{array}\right.
$$

Substituting expressions $x_{k}\left(t_{j-1}-0\right), j=\overline{2, M+1}, k=\overline{1, N}$ into the right-hand side of equations (15) we obtain

$$
\begin{gathered}
x_{k}(t)=\left[\left(x_{k}\left(t_{j-2}\right)\right) \exp \left\{\int_{t_{j-2}}^{t_{j-1}} b(s) y(s) d s\right\}+\right. \\
\left.+\int_{t_{j-2}}^{t_{j-1}} a_{k}(\tau) y(\tau) \exp \left\{\int_{\tau}^{t_{j-1}} b(s) y(s) d s\right\} d \tau+\zeta_{j-1, k}\right] \exp \left\{\int_{t_{j-1}}^{t} b(s) y(s) d s\right\}+ \\
+\int_{t_{j-1}}^{t} a_{k}(\tau) y(\tau) \exp \left\{\int_{\tau}^{t} b(s) y(s) d s\right\} d \tau, t \in\left(t_{j-1}, t_{j}\right), j=\overline{2, M+1}, k=\overline{1, N} .
\end{gathered}
$$

Using similar considerations we have the expressions

$$
\begin{gathered}
x_{k}(t)=\left[\ldots \left[x_{k}^{0} \exp \left\{\int_{t_{0}}^{t_{1}} b(s) y(s) d s\right\}+\right.\right. \\
\left.+\int_{t_{0}}^{t_{1}} a_{k}(\tau) y(\tau) \exp \left\{\int_{\tau}^{t_{1}} b(s) y(s) d s\right\} d \tau+\zeta_{1, k}\right] \times \ldots \times \exp \left\{\int_{t_{j-2}}^{t_{j-1}} b(s) y(s) d s\right\}+ \\
\left.+\int_{t_{j-2}}^{t_{j-1}} a_{k}(\tau) y(\tau) \exp \left\{\int_{\tau}^{t_{j-1}} b(s) y(s) d s\right\} d \tau+\zeta_{j-1, k}\right] \times \exp \left\{\int_{t_{j-1}}^{t} b(s) y(s) d s\right\}+
\end{gathered}
$$




$$
+\int_{t_{j-1}}^{t} a_{k}(\tau) y(\tau) \exp \left\{\int_{\tau}^{t} b(s) y(s) d s\right\} d \tau, t \in\left(t_{j-1}, t_{j}\right), j=\overline{1, M+1}, k=\overline{1, N} .
$$

The theorem has been proven.

Corollary 1. Let the parameters of the system (14) be constant functions on the intervals $t \in\left(t_{j-1}+0, t_{j}-0\right), j=\overline{1, M+1}$ (the piecewise constant functions) and the conditions $a_{j, k}=a_{k}(t), b_{j}=b_{j, k}(t), j=\overline{1, M+1}, k=\overline{1, N}$ be satisfied. The functions $x_{k}(t), k=\overline{1, N}$, $t \in\left(t_{0}, T\right)$ can be represented as

$$
\begin{gathered}
x_{k}(t)=\left(\left(\ldots \left(x_{k}^{0} \frac{\left(a_{1}-y\left(t_{0}\right) b_{1}\right) \exp \left\{\left(t_{0}-t_{1}\right) a_{1}\right\}+y\left(t_{0}\right) b_{1}}{a_{1} \exp \left\{\left(t_{0}-t_{1}\right) a_{1}\right\}}+\right.\right.\right. \\
\left.\left.+\frac{a_{1, k} y\left(t_{0}\right)\left(1-\exp \left\{\left(t_{0}-t_{1}\right) a_{1}\right\}\right)}{a_{1} \exp \left\{\left(t_{0}-t_{j_{1}}\right) a_{1}\right\}}+\zeta_{1, k}\right) \times \ldots\right) \times \\
\times \frac{\left(a_{j-1}-y\left(t_{j-2}\right) b_{j-1}\right) \exp \left\{\left(t_{j-2}-t_{j-1}\right) a_{j-1}\right\}+y\left(t_{j-2}\right) b_{j-1}}{a_{j-1} \exp \left\{\left(t_{j-2}-t_{j-1}\right) a_{j-1}\right\}}+ \\
\left.+\frac{a_{j-1, k} y\left(t_{j-2}\right)\left(1-\exp \left\{\left(t_{j-2}-t_{j-1}\right) a_{j-1}\right\}\right)}{a_{j-1} \exp \left\{\left(t_{j-2}-t_{j_{1}}\right) a_{j-1}\right\}}+\zeta_{j-1, k}\right) \times \\
\times \frac{\left(a_{j}-y\left(t_{j-1}\right) b_{j}\right) \exp \left\{\left(t_{j-1}-t\right) a_{j}\right\}+y\left(t_{j-1}\right) b_{j}}{a_{j} \exp \left\{\left(t_{j-1}-t\right) a_{j}\right\}}+ \\
+\frac{a_{j, k} y\left(t_{j-1}\right)\left(1-\exp \left\{\left(t_{j-1}-t\right) a_{j}\right\}\right)}{a_{j} \exp \left\{\left(t_{j-1}-t\right) a_{j}\right\}}, t \in\left(t_{j-1}, t_{j}\right), j=\overline{1, M+1}, k=\overline{1, N},
\end{gathered}
$$

where $a_{j}=\sum_{i=1}^{N} a_{j, i}+b_{j} L, j=\overline{1, M+1}$.

4. Results of numerical experiment. Analysis of model with non-stationary parameters of spreading of one type of information is given as an example.

Let us consider the special case of the model (3)

$$
\dot{x}=(a(t)+b(t) x(t))(L-x(t)), t \in\left(t_{0}, T\right), x\left(t_{0}\right)=x^{0} \in R^{1}, k=\overline{1, N} .
$$

Suppose that at the points $t_{0}<t_{1}<\cdots<t_{M}<t_{M+1}=T, t_{j} \in\left(t_{0}, T\right), j=\overline{1, M}$ the function $a(t), t \in\left[t_{0}, T\right]$ is observed

$$
\tilde{a}_{i}=a\left(t_{i}\right), i=\overline{0, M+1} .
$$

Then instead of analysis of the system (17) we investigate the next model

$$
\left[\begin{array}{l}
\dot{\hat{x}}=(\hat{a}(t)+b(t) \hat{x}(t))\left(L-\hat{x}_{j}(t)\right), t \neq t_{j}, j=\overline{1, M}, t \in\left(t_{0}, T\right), \\
x\left(t_{j}\right)=x\left(t_{j}-0\right)+\zeta_{j}, j=\overline{1, M}, \\
x\left(t_{0}\right)=x^{0}
\end{array}\right.
$$

where

$$
\hat{a}(t)=\frac{\tilde{a}_{j}+\tilde{a}_{j+1}}{2}, \quad t \in\left(t_{j-1}, t_{j}\right), \quad j=\overline{1, M+1}, \quad \hat{a}\left(t_{0}\right)=a\left(t_{0}\right) .
$$

Thus the solution of the system (19) is the approximate solutions of the system (17).

Let the parameters of the model satisfy $(17)$, if $L=100, x^{0}=10, a(t)=0.5 \sin (3 t)$, $b(t)=0.003, t \in(0 ; 5), \Delta t=t_{j+1}-t_{j}=0.5, j=\overline{0 ; 10}$.

We obtain the function approximation of the parameter $a(t), t \in(0 ; 5)$ (Fig. 1.). Then on the basis the formula (16) we get the approximate solutions of the equation (17) (Fig. 2).

The analysis of these results has demonstrated the practical meaning to finding approximate solutions of the non-linear differential equations with non-stationary parameters describing the information spreading processes with jump discontinuity. 


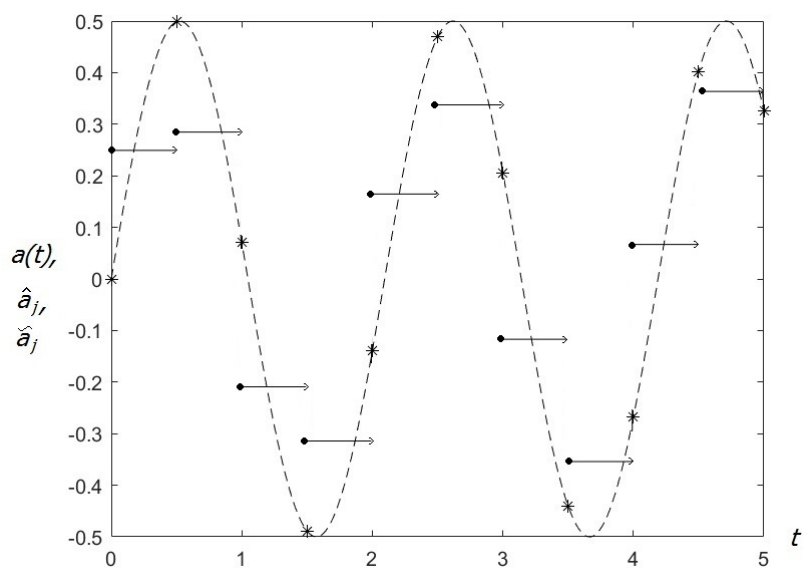

Figure 1: Dynamics of the parameter $a(t), t \in(0,5)$ of the system $(3)$, where dotted line is $a(t), t \in(0,5)$, hull line is $\hat{a}_{j}, j=\overline{1 ; 10}$, symbols $*$ is $\tilde{a}_{i}, i=\overline{0 ; 11}$.

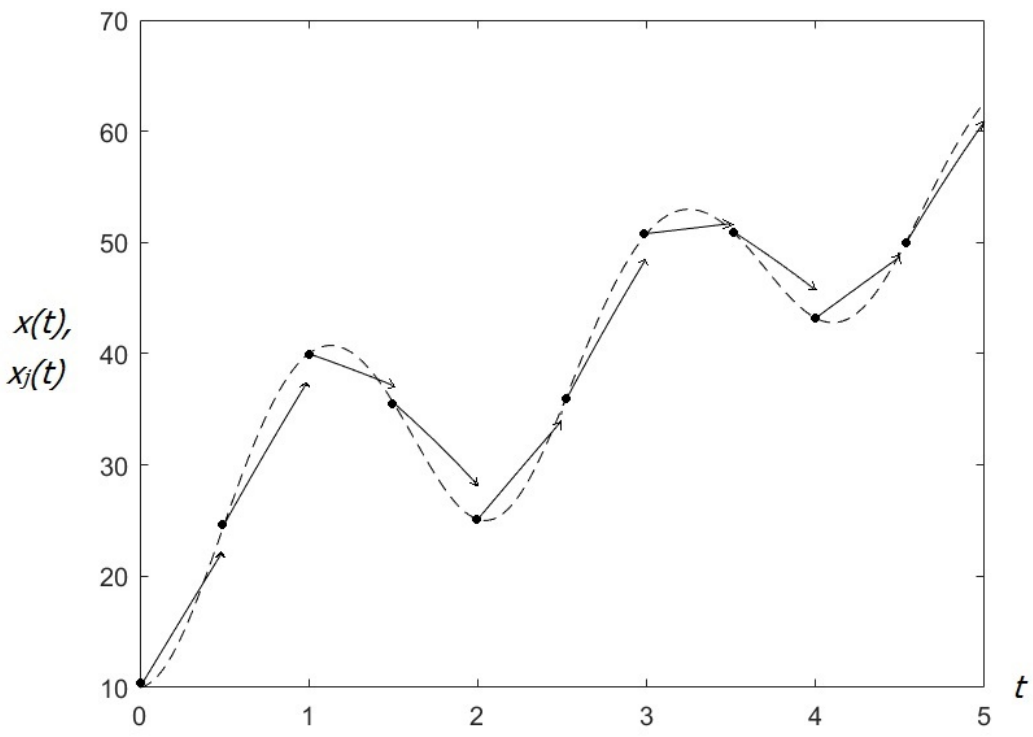

Figure 2: Dynamics of the system (17) and (19), where dotted line is $x(t), t \in(0,5)$, full line is $\hat{x}(t), t \in(0,5)$. 


\section{REFERENCES}

1. Lopushanska H.P., Buhrii O.M., Lopushanskyj A.O., Differential equations and equations of mathematical physics, Publisher I.E. Chychykov, Lviv, 2012. (in Ukrainian)

2. Samoilenko A.M., Perestyuk N.A., Parasyuk I.O., Differential equations, Kyiv: Lybid, 2003. (in Ukrainian)

3. Bokalo M.M., Tsebenko A.M. Optimal resource coefficient control in a dynamic population model without initial conditions// Visnyk of the Lviv Univ. Series Mech. Math. - 2016. - V.81. - P. 39-57.

4. Mikhailov A.P., Marevtseva N.A., Models of Information Warfare// Mathematical Models and Computer Simulations. - V.4, №3. - 2012. - P. 251-259.

5. Nakonechnyi O.G., Zinko P.M. Confrontation problems with the dynamics Gompertzian systems// Journal of Computational and Applied Mathematics. - 2015. - №3(120). - P. 50-60. (in Ukrainian)

6. Nakonechnyi O.G., Shevchuk I.M. Mathematical model of information spreading process with nonstationary parameters// Bulletin of Taras Shevchenko National University of Kiev. Series Physics and Mathematics. - 2016. - №3. - P. 98-105. (in Ukrainian)

7. Shevchuk I.M. Stability of solutions of mathematical models of information spreading process with external control// Journal of Computational and Applied Mathematics. - 2017. - №1. - P. 99-111. (in Ukrainian)

8. Nakonechnyi O., Shevchuk I. Stability under stocgastic perturbation of solutions of mathematical models of information spreading process with external control// Mathematical Modeling and Computing. - V.5, №1. - 2018. - P. 66-73.

9. Samoilenko A.M., Perestyuk N.A., Impulsive differential equations, Singapore: World Scientific, 1995.

Taras Shevchenko National University of Kyiv

Department of System Analysis and Decision Making Theory

Kyiv, Ukraine

a.nakonechniy@gmail.com

petro.zinko@gmail.com

shevchuk.juli@ukr.net 